\title{
Ansiedade na Performance Musical de Música de Câmara O Efeito do Biofeedback Como Medida Interventiva
}

\author{
Samuel Barros, ${ }^{1}$ Helena Marinho, ${ }^{2}$ Anabela Pereira, ${ }^{3}$ \\ Ana Ribeiro, ${ }^{4}$ Isabel Souto, ${ }^{5}$ Luis Sancho ${ }^{6}$
}

\begin{abstract}
RESUMO
A Ansiedade na Performance Musical (APM) é descrita como uma apreensão persistente que compromete as competências performativas, tanto em performances a solo como em grupo. Para além dos sintomas fisiológicos, como aumento do batimento cardíaco, hiperventilação, transpiração ou náusea, está associada a sintomas de natureza psicológica e cognitiva de impacto negativo na performance. Este trabalho pretende estudar a eficácia do treino de biofeedback em contexto de performance de música de câmara. Com um desenho do tipo quasi - experimental, foram selecionados dois quartetos de trombonistas, sendo o quarteto (1) o grupo experimental e o quarteto (2) o grupo de controle. Ambos realizaram apresentações públicas, antes e depois da intervenção. O quarteto (1) foi submetido a um programa de oito sessões bissemanais de treino de biofeedback, no intuito de verificar a sua contribuição para diminuir os efeitos da APM. Na avaliação da eficácia da intervenção foram aplicados pré e pós-intervenção os questionários State Trait Anxiety Inventory (STAI), Kenny Music Performance Anxiety Inventory (K-MPAI) e Kessler Psychological Distress Scale (K10). Os resultados sugerem que este tipo de intervenção revela-se favorável no que diz respeito à diminuição dos níveis de APM em contexto de música de câmara. São referidas algumas implicações deste estudo para a redução da ansiedade de performance quer em contextos de formação e ensino da música quer em contextos de prática profissional.
\end{abstract}

Palavras-chave: Ansiedade na performance musical. Música de câmara. Biofeedback. Trombone.

MUSICAL PERFORMANCE ANXIETY OF CHAMBER MUSIC: THE EFFECT OF BIOFEEDBACK AS AN INTERVENTIONAL MEASURE

\section{ABSTRACT}

Music Performance Anxiety (MPA) is described as a persistent apprehension that compromises performative skills, both in solo or group performances. In addition to physiological symptoms, such as increased heart rate, hyperventilation, sweating or nausea, MPA is associated with psychological and cognitive symptoms of performance negative impact. The present work intends to study the effectiveness of biofeedback training in the context of chamber music performance, applying a quasi-experimental. 2 quartets of trombonists were selected, quartet (1) being the experimental group and quartet (2) the control group. Both made public presentations, before and after the intervention. Quartet (1) underwent a program of 8 biweekly sessions of biofeedback training, in order to verify if it contributed to diminish the effects of MPA. The questionnaires State Trait Anxiety Inventory (STAI), Kenny Music Performance Anxiety Inventory (K-MPAI) and Kessler Psychological Distress Scale were used to evaluate the intervention efficacy. The results suggest that this type of intervention is favorable with regard to the decrease of APM levels in the context of chamber music. Some implications of this study are mentioned for the reduction of performance anxiety in training and teaching contexts, as well in professional musical practice.

Keywords: Music performance anxiety. Chamber music. Biofeedback. Trombone.

Recebido em: 18/3/2019

Aceito em: $9 / 5 / 2019$

\footnotetext{
Doutorando em Música (Estudos em Performance), Mestre em Música (Performance) pela Universidade de Aveiro, Portugal. Licenciado em Música pela Universidade Estadual do Ceará (Uece). Desenvolve investigações sobre a ansiedade na performance musical no contexto da educação e ensino da música, com colaboração nas atividades no âmbito dos projetos de investigação do StressLab ${ }^{\circledR}$ (Laboratório de Estudos de Investigação e Intervenção no Stresse), com particular relevo no treino das sessões de Biofeedback para estudantes/profissionais de música e professores do Ensino Superior. samuellbarrim@hotmail.com Doutora em Música pela University of Sheffield, Mestre em música pela University of Kansas e Licenciada em Línguas e Literaturas Modernas pela Universidade do Porto. Professora-auxiliar do Departamento de Comunicação e Arte da Universidade de Aveiro, Inet-md. helena.marinho@ua.pt

${ }^{3}$ Doutora em Psicologia pela University of Hull, United kingdom, Licenciada e Mestre em Psicologia pela Universidade de Coimbra. Docente na área da Psicologia da Educação na interface da psicologia da saúde (Departamento de Educação e Psicologia da Universidade de Aveiro). anabelapereira@ua.pt

${ }^{4}$ Mestre em Psicologia da Saúde e Reabilitação Neuropsicológica e Licenciada em Psicologia (Universidade de Aveiro). Exerce Psicologia no âmbito Educacional como Psicóloga Estagiária num Agrupamento escolar, correspondente ao seu ano profissional júnior na Ordem dos Psicólogos Portugueses.isabel.souto@ua.pt

${ }^{5}$ Licenciada e mestre em Psicologia pela Universidade de Aveiro e investigadora colaboradora no Centro de Investigação Didática e Tecnologia na Formação de Formadores (CIDTFF) da Universidade de Aveiro. ana.maria.ferreira@ua.pt

${ }^{6}$ Doutor em Psicologia pela Universidade de Aveiro, Licenciado e Mestre em Ciências Biomédicas pelo King's College, University of London. Professor-adjunto na Escola Superior de Saúde da Universidade de Aveiro. Isancho@ua.pt
} 


\section{INTRODUÇÃO}

A ansiedade na performance é descrita pelos músicos como uma persistente e angustiante apreensão que compromete as competências performativas em público. No que respeita à Ansiedade na Performance Musical (APM), esta pode acontecer com cantores ou qualquer instrumentista, tanto em performances a solo como em grupo (BARBAR; CRIPPA; OSÓRIO, 2014). A ocorrência de APM varia de acordo com o contexto em que os performers estão inseridos, sendo possível identificar que os ensaios, as performances a solo ou em grupo, as audições públicas e outras, são passiveis de desencadear APM (KENNY, 2005).

A APM surge como uma manifestação emocional pluridimensional que pode incluir um conjunto de respostas cognitivas (sensação de medo, desastre ou de se atrapalhar consigo mesmo, pensamentos automáticos negativos sobre falhas de memória, erros de notas, diminuição da concentração), comportamentais (postura retraída, falhas técnicas e tremores) e fisiológicas (hiperventilação, salivação, aceleração dos batimentos cardíacos, problemas gastrointestinais, liberação excessiva de adrenalina e cortisol) (KENNY, 2011, 2016), que podem acarretar prejuízos no desempenho da performance em público, com impacto profissional e pessoal indissociável.

Diversos estudos têm sido realizados no sentido de dar resposta apropriada a esta problemática, denotando-se a necessidade de medidas interventivas adequadas à prevenção e combate da APM (KENNY, 2011; RAY, 2014; SINICO; WINTER, 2013), contribuindo assim para o bem-estar dos estudantes e profissionais que sofrem com essa condição.

É nesse sentido que, de uma forma geral, o biofeedback constitui-se como uma técnica não invasiva, composto por um conjunto de sensores não intrusivos, que permitem a captação e recolha de sinais à superfície da pele (atividade muscular, temperatura, atividade eletrodérmica, respiração, ritmo cardíaco, pressão arterial, atividade elétrica do cérebro e/ou corrente sanguínea). Estes sinais são filtrados, amplificados, digitalizados e transmitidos via Bluetooth ${ }^{\circledR}$ para um computador no qual são representados em tempo real por meio de valores numéricos, gráficos, imagens ou sons (CHALÓ et al., 2017b; COSTA, 2016; SANCHO, 2013). Por intermédio deste feedback são permitidas ao indivíduo a identificação e a tomada de consciência das reações psicofisiológicas inerentes a um estado de ansiedade (tensão dos músculos, temperatura da pele, frequência cardíaca e alterações na circulação sanguínea), induzindo-o a aprender a alterá-las de forma voluntária (CHALÓ et al., 2016, 2017b; COSTA, 2016; SANCHO, 2013). Este maior entendimento traduz-se no aumento da confiança e controle das respostas ansiosas de forma a substituí-las por outras mais adaptativas antes ou durante situações emocionalmente exigentes (CHALÓ et al., 2016, 2017a; COSTA, 2016; JARASIUNAITE et al., 2015; KASSEL; LEMAY, 2012; SANCHO, 2013; SCHWARTZ; ANDRASIK, 2016; WEST, 2007).

Estudos recentes adotam o biofeedback como modelo interventivo no autocontrole da sintomatologia psicofisiológica decorrente da ansiedade e distress, evidenciando-se como uma terapêutica eficaz (CHALÓ et al., 2016, 2017a; COSTA, 2016; SANCHO, 2013).

Os programas de intervenção com biofeedback variam de acordo com os níveis de ansiedade, os treinos realizados e as diferentes áreas de estudo. Neste sentido Chaló et al. (2016) constataram a eficácia do biofeedback em contexto universitário, e demostraram a sua eficiência a partir de cinco sessões, destacando-se que em oito sessões bissemanais obtiveram um resultado de $100 \%$ em relação à diminuição da ansiedade. O biofeeback também se mostrou eficaz aplicado de forma exclusiva, ou em comparação com outras técnicas de intervenção (CHALÓ; BATISTA; PEREIRA, 2017).

Em particular no contexto musical, esta ferramenta tem sido utilizada para diminuir a tensão dos músculos da face em instrumentistas de sopro e metais, dos músculos do antebraço de violinistas e clarinetistas, bem como músculos do polegar de violinistas e violistas (LEHMANN; SLOBODA; WOODY, 2007). Segundo Thurber et al. (2010), a eficácia do treino com o biofeedback é variável de acordo com os níveis de ansiedade, existindo uma variabilidade de pelo menos quatro sessões de 30 a 50 minutos para demonstrar eficácia contra os efeitos da ansiedade. Ressalva-se, no entanto, que existe um número limitado de estudos direcionados à investigação desta técnica como adequada à intervenção na APM (KENNY, 2005).

Por essa razão, o presente estudo pretende investigar os comportamentos psicofisiológicos em contexto de performance de música de câmara de um quarteto de trombonistas, nomeadamente numa audição pública, bem como estudar a diminuição da sintomatologia ansiosa, especificamente ao nível da eficácia do treino de biofeedback neste contexto.

\section{MÉTODO}

Foi conduzido um estudo quasi-experimental, para o qual foram selecionados dois quartetos de trombonistas, sendo o quarteto (1) o grupo experi- 
mental e o quarteto (2) o grupo de controle. O quarteto 1 realizou o programa de biofeedback e o quarteto 2 não foi alvo de qualquer tipo de intervenção. Ambos realizaram apresentações públicas, antes e depois da aplicação do programa de intervenção, sendo avaliados em ambos os momentos por meio de inventários específicos, tendo os resultados sido comparados. Foi oferecida a possibilidade de realizar o programa de biofeedback a todos os participantes do grupo de controle, após o término do estudo.

\section{Participantes}

Participaram deste estudo oito estudantes de Música da Universidade de Aveiro, todos alunos da classe de trombone, com idades compreendidas entre 19 e 23 anos ( $M=20.63$; $D P=1.41)$.

A amostra foi constituída por seis participantes do sexo masculino (75\%) e dois do sexo feminino (25\%), sendo $50 \%$ do grupo experimental $(n=4)$ e $50 \%$ do grupo de controle $(n=4)$. As duas participantes do gênero feminino foram distribuídas por cada um dos grupos.

Os participantes integram o curso de Licenciatura em Música na variante de performance (75\%; $\mathrm{n}=6$ ) (primeiro, segundo e terceiro anos), e Mestrado em Ensino da Música (25\%; $n=2$ ). Ressalva-se que os mestrandos concluíram a Licenciatura nesta mesma universidade, também na variante performance.

Nenhum participante estava em processo psicoterapêutico e nem sujeitos à medicação psicofarmacológica. Todos colaboraram de forma voluntária, e assinaram um consentimento no qual foram informados dos objetivos e uso posterior dos dados recolhidos, pelo que foram respeitados os princípios éticos e deontológicos inerentes ao desenvolvimento de uma investigação.

\section{Materiais}

\section{Inventário de Ansiedade de Performance Musical de Kenny (K-MPAI)}

O K-MPAI (KENNY, 2009, versão para língua portuguesa de ROCHA; DIAS-NETO; GATTAZ, 2011) é um questionário que permite avaliar a presença de ansiedade em performance musical, considerando que esta pode ter origem em diferentes componentes. Assim, o K-MPAl contém informações referente à história pessoal, parental e da infância, e examina igualmente os sinais associados à ansiedade na performance musical, nomeadamente os pensamentos negativos, tensão muscular e alterações de memória. Esse questio- nário contém 40 itens, cujo preenchimento se dá por meio de uma escala de Likert com 7 opções de respostas, as quais pontuam entre -3 , que revela uma opinião de "discordar plenamente", a +3 , que representa uma opinião de "concordar plenamente". As pontuações totais variam entre 0 e 240 , observando-se que uma alta pontuação significa um alto nível de ansiedade na performance musical (KENNY, 2009). A análise de consistência interna para a versão traduzida para o Português revelou-se boa, com bons níveis de Alpha de Cronbach $(\alpha)=0.957$ (ROCHA, 2012). Para este estudo verificou-se no primeiro e no segundo momento $\alpha=0.92$ e $\alpha=0.94$, respectivamente.

\section{O State-Trait Anxiety Inventory (STAI-Y)}

O STAI (SPIELBERGER, 1983, versão portuguesa de SANTOS; SILVA, 1997), é um instrumento amplamente utilizado para avaliação do nível geral de ansiedade. É um questionário de autorresposta composto por 40 questões, divididas em duas subescalas de 20 itens cada, que permitem identificar a presença de ansiedade-estado e ansiedade-traço (STAI-Y-1 e STAI-Y-2). A ansiedade-estado está relacionada com situações específicas (momento de uma audição ou performance pública), enquanto a ansiedade-traço define de forma permanente o indivíduo, ou seja, é intrínseca a ele(a). Para cada item o inquirido registra a sua resposta numa escala de Likert de 4 níveis, em função da sua intensidade (no STAI-Y-1- "nada", "um pouco", "moderadamente", "muito"), e a sua frequência (no STAI-Y-2 - "quase nunca", "algumas vezes", "frequentemente", "quase sempre"). A amplitude dos resultados por subescala varia entre 20 e 80 , sendo considerado que pontuações acima de 40 (por subescala) corresponde à presença de sintomas clinicamente significativos.

O estudo de adaptação para a população portuguesa reflete bons níveis de consistência interna, com $\alpha=0,85$ e $\alpha=0,86$ no STAI-Y-1 e STAI-Y-2, respectivamente (SANTOS; SILVA, 1997). No presente trabaIho os índices de consistência interna para o STAI-Y-1 corresponderam a $\alpha=0.73$ e $\alpha=0.72$, para o primeiro e segundo momento, respectivamente. Para o STAI-Y-2 foi obtido $\alpha=0,94$ (aplicado num único momento).

\section{Kessler Psychological Distress Scale (K10)}

O K10 (KESSLER et al., 2002, versão portuguesa por PEREIRA et al., 2017) é uma escala de triagem de 10 questões que permite avaliar os sintomas de desconforto psicológico não específicos em estudantes universitários e trabalhadores em geral, remeten- 
do para os últimos 30 dias dos inquiridos. A medida consiste em autorrelato baseado em questões sobre ansiedade e depressão, respondidas numa escala de Likert de 1 a 5 pontos ("nenhum dia", "poucos dias", "alguns dias", "a maior parte dos dias" e "todos os dias"). A amplitude dos resultados por subescala varia entre 10 e 50, considerando-se que a obtenção de pontuações acima de 22 correspondem ao risco de desenvolvimento de sintomatologia clinicamente significativa (PEREIRA et al., 2017).

$\mathrm{O}$ estudo de adaptação para a população portuguesa apresentou boa consistência interna com $\alpha=0,910$. No presente estudo verificou-se para o primeiro e segundo momento $\alpha=0,63$ e $\alpha=0,82$, respectivamente.

\section{Biofeedback 2000-pert}

O Biofeedback $2000^{x-p e r t}$ é um equipamento portátil, desenvolvido pela SCHUHFRIED GmbH, que cumpre as normas europeias para dispositivos médicos, garantindo a precisão e validade no seu funcionamento.

Trata-se de um sistema composto por diversos módulos, cada um destinado a captar dados fisiológicos específicos. Os módulos deste sistema recolhem sinais à superfície da pele, por meio de sensores não intrusivos, que são posteriormente transmitidos via Bluetooth ${ }^{\circledR}$ para um computador no qual, por meio do seu software específico, são interpretados e representados de diversas formas de acordo com o programa selecionado. Os módulos caracterizam-se pela sua pequena dimensão e leveza que, associadas à utilização do Bluetooth ${ }^{\circledR}$ para transmissão de dados, permitem uma maior liberdade de movimentos por parte do utilizador.

No presente estudo foi utilizado o módulo MUL$\mathrm{TI}$, composto por sensores que recolhem sinais à superfície da pele de atividade eletrodérmica (EDA), temperatura corporal (TEMP), pulsação (PULS) e aumento da frequência de movimento (MOT).

A colocação dos sensores foi efetuada de acordo com o manual do equipamento, nomeadamente na mão não dominante, o sensor EDA na segunda falange do dedo médio, o sensor TEMP colocado na primeira falange do dedo médio e o sensor PULS na primeira falange do dedo indicador.

\section{Procedimento}

Este estudo foi realizado durante o ano letivo $2017 / 2018$, tendo em sua primeira fase (pré-intervenção com biofeedback) o recolhimento das informações sociodemográficas e dos questionários STAI-Y-1 e STAI-Y-2, K-MPAI e K10.
As sessões de biofeedback tiveram lugar no StressLab do Departamento de Educação e Psicologia da Universidade de Aveiro. A intervenção foi operacionalizada bissemanalmente durante 4 semanas, perfazendo um total de 8 sessões de 15 minutos cada.

Para a segunda fase (pós-intervenção com biofeedback) os participantes foram novamente avaliados com a recolha dos questionários STAI-Y-1, K-MPAI e K10, no momento prévio à situação de audição públi$\mathrm{ca}$, por inquéritos 30 minutos antes. Posteriormente foi realizada uma análise comparativa de resultados.

\section{Análises Estatísticas}

Os dados foram sujeitos à análise estatística com o software IBM ${ }^{\circledR}$ SPSS ${ }^{\circledR}$ Statistics, versão 25 , de forma a aferir a eficácia do programa de biofeedback e proceder à análise da estatística descritiva e inferencial consoante os objetivos e variáveis propostos neste estudo. Admitiu-se, portanto, como variáveis dependentes a ansiedade associada à performance, ansiedade-estado e distress, e, como independente, o programa de intervenção com biofeedback. Calculou-se o Alpha de Cronbach $(\alpha)$, de forma a avaliar a consistência interna das escalas utilizadas para a amostra em estudo, e, considerando que a amostra apresenta um tamanho reduzido, verificou-se que as variáveis respeitam os pressupostos da normalidade, admitindo-se a utilização de testes paramétricos, pelo que foram utilizados testes t-emparelhados.

\section{RESULTADOS}

Para este estudo observou-se que a diferença da pontuação média na ansiedade-traço entre homens ( $M=37.16$; $D P=4.84)$ e mulheres $(M=61.00$; $\mathrm{DP}=12.73$ ) é significativo [t(6)=- 4.28; $\rho<0.05]$. As muIheres, portanto, demonstram ser mais ansiosas do que os homens (Figura 1).

Figura 1 - Valores da Média do fator da ansiedade-traço dos participantes do sexo masculino e feminino da população amostral

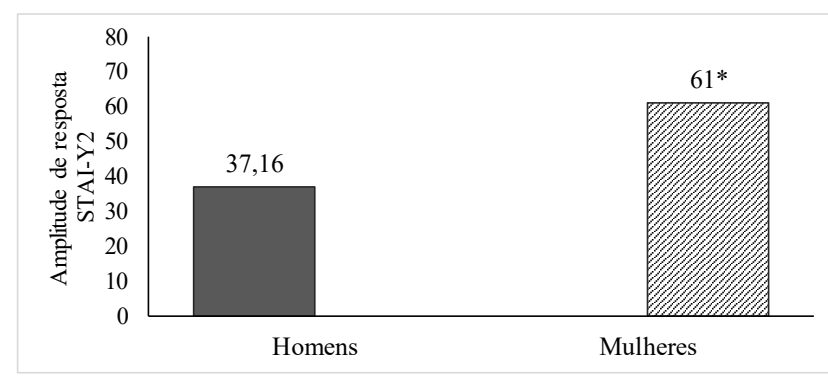

$\rho<0.05$.

Fonte: Dados da pesquisa. 
Relativamente aos resultados obtidos pelos indivíduos do grupo experimental no primeiro momento $(M=49.75 ; D P=4.72)$ e no segundo momento ( $M=37.50$; $D P=2.38$ ) por meio do STAI-Y-1, observou-se uma redução estatisticamente significativa no grau de ansiedade-estado [t(3)=5.96; $\rho<0.05]$ (Figura 2).

Figura 2 - Valores da Média do fator da ansiedade-estado dos participantes do grupo experimental antes e depois

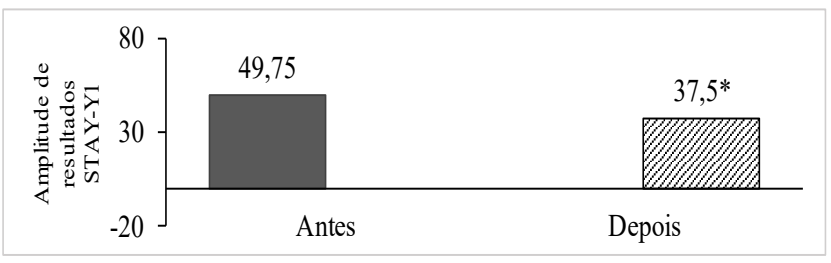

$\rho<0.05$

$$
\text { Fonte: Dados da pesquisa. }
$$

No que respeita aos resultados obtidos pelos participantes no K-MPAI $[\mathrm{t}(3)=2.29 ; \rho=0.11]$ e no Distress $[\mathrm{t}(3)=0.84 ; \rho=0.46]$ não se verificaram, por via do teste t-emparelhados, diferenças estatisticamente significativas para os resultados obtidos pelo quarteto 1 depois das sessões de biofeedback.

Para o quarteto 2 observou-se que a diferença entre a pontuação calculada por meio do Distress, K-MPAI e STAI-Y-1 no primeiro e segundo momento não é significativa, respectivamente $[\mathrm{t}(3)=0 ; \rho=1]$, $[\mathrm{t}(3)=0.64 ; \rho=0.57]$ e $[\mathrm{t}(3=-0.57 ; \rho=0.61]$. Conclui-se que os fatores de ansiedade avaliados com estas escalas não possuem diferenças significativas em ambos os momentos.
Comparando as médias de todas as escalas de medição, observou-se que o quarteto 1 antes das sessões de biofeedback mostrou-se com níveis de estresse e ansiedade maiores do que o quarteto 2 (Quadro 1). Embora se tenha notado que após as sessões de biofeedback estes níveis tenham diminuído, o quarteto 1 ainda permaneceu com os níveis maiores, mesmo sendo por uma discreta diferença (Quadro 1).

\section{DISCUSSÃO}

Este estudo teve como objetivo verificar a eficácia do treino de biofeedback na redução da ansiedade na performance musical em contexto de música de câmara.

Os resultados permitem concluir que, no que se relaciona à ansiedade-traço, na amostra do estudo em questão as mulheres são predispostas a serem mais ansiosas do que os homens. Este resultado foi também encontrado em Osborne, Kenny e Holsomback (2005). Embora haja algumas pesquisas que apontem que os homens apresentam mais ansiedade do que as mulheres (OREJUDO et al., 2017) e outras que referem níveis de ansiedade iguais para ambos os sexos (FEHM; SCHMIDT, 2006), a maioria das pesquisas aponta as mulheres como sendo mais propensas a experimentar altos níveis de ansiedade na performance, independentemente da idade dos indivíduos (OSBORNE; KENNY, 2008; PAPAGEORGI, 2007).

No que diz respeito à eficácia do biofeedback na redução dos níveis de estresse e ansiedade na performance musical sentida pelos participantes desta investigação, é importante destacar que foi verificada

Quadro 1 - Comparação entre grupos da média antes e depois da intervenção com biofeedback

\begin{tabular}{|c|c|c|c|c|c|c|c|c|}
\hline \multirow{2}{*}{\multicolumn{2}{|c|}{ Grupo }} & \multirow{2}{*}{ Valores } & \multicolumn{2}{|c|}{ Distress } & \multicolumn{2}{|c|}{ K-MPAI } & \multicolumn{2}{|c|}{ Ansiedade-estado } \\
\hline & & & Antes & Depois & Antes & Depois & Antes & Depois \\
\hline \multirow{4}{*}{$\begin{array}{l}\overrightarrow{0} \\
\frac{0}{0} \\
\frac{0}{0} \\
\vec{z}\end{array}$} & \multirow{4}{*}{ 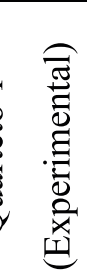 } & $\mathrm{M}$ & 20.50 & 19.25 & 126.00 & 100.25 & 49.75 & 37.50 \\
\hline & & DP & 2.89 & 3.40 & 40.85 & 40.01 & 4.72 & 2.38 \\
\hline & & Mín. & 17.00 & 16.00 & 94.00 & 52.00 & 43.00 & 36.00 \\
\hline & & Máx. & 24.00 & 24.00 & 183.00 & 150.00 & 53.00 & 41.00 \\
\hline \multirow{4}{*}{ 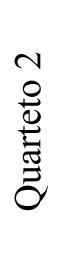 } & & $\mathrm{M}$ & 17.50 & 17.50 & 110.75 & 98.25 & 40.00 & 41.75 \\
\hline & 음 & DP & 3.32 & 5.26 & 24.5 & 39.20 & 5.72 & 5.00 \\
\hline & : & Mín. & 13.00 & 12.00 & 83.00 & 53.00 & 34.00 & 35.00 \\
\hline & & Máx. & 21.00 & 22.00 & 138.00 & 142.00 & 47.00 & 46.00 \\
\hline
\end{tabular}

Fonte: Dados da pesquisa. 
uma diminuição na média dos níveis de ansiedade e estresse de todas as escalas, nomeadamente STAI-Y-1, K-MPAI e K10. Embora após o treino de biofeedback o resultado tenha sido não significativo estatisticamente para o K-MPAI e o K10, para o STAI-Y-1 o resultado mostrou ser estatisticamente significativo após os treinos, verificando-se diminuição da ansiedade-estado. Com isso, confirmamos a pesquisa de West (2007), que sugere que o treino de biofeedback é capaz de equilibrar o corpo e a mente, permitindo maior consciência e concentração para a tarefa que Ihe é atribuída. Do mesmo modo, é permitido ao indivíduo a autorregulação das suas sensações fisiológicas (SCHWARTZ; ANDRASIK, 2016; WEST, 2007).

Apesar dos resultados terem se revelado favoráveis na diminuição da APM, ressalva-se que esta diferença não foi estatisticamente significativa em todos os fatores avaliados. É importante destacar, contudo, o trabalho de Thurber et al. (2010), no qual os resultados de treino de biofeedback revelou ter impacto significativo na redução dos efeitos da APM em estudantes de música, em sintonia com os resultados de Chaló et al. (2016), em contexto universitário.

\section{CONCLUSÃO}

Tendo em consideração os objetivos do presente estudo, o treino de biofeedback revelou-se uma técnica com potencial aplicabilidade no combate à ansiedade na performance musical em contexto de música de câmara. Conscientes das limitações inerentes a este tipo de estudo (principalmente pelo tamanho reduzido da amostra), sugerimos a realização de novos estudos com amostras de maior dimensão e para outras formações musicais.

Além disso, importa o aprofundamento desta temática visando a compreender de forma empírica a eficácia do biofeedback para a redução da ansiedade de performance, quer em contextos de formação e ensino da música, quer em contextos de prática profissional.

\section{REFERÊNCIAS}

BARBAR, A. E.; CRIPPA, J. A.; OSÓRIO, F. L. Parameters for screening music performance anxiety. Revista Brasileira de Psiquiatria, v. 36, n. 3, p. 245-247, set. 2014.

CHALÓ, P. et al. Biofeedback and anxiety in higher education: comparison of two brief intervention programs. Psicologia, Saúde \& Doenças, v. 17, n. 1, p. 60-66, mar. 2016.

CHALÓ, P. et al. Gestão da ansiedade na adaptação ao ensino superior: o contributo do biofeedback. Revista da Associação Portuguesa de Psicologia, v. 31, n. 2, p. 137-141, 2017a.
CHALÓ, P.; BATISTA, P.; PEREIRA, A. Biofeedback training on university student's anxiety management: A systematic review. Biomedical Research and Clinical Practice, v. 2, n. 1, p. 1-6, 2017.

CHALÓ, P. et al. Brief biofeedback intervention on anxious freshman university students. Applied Psychophysiology and Biofeedback, v. 42, n. 3, p. 163-168, 19 set. 2017b.

COSTA, V. A. R. Impacto do biofeedback nos níveis de stress, ansiedade e autorregulação emocional em estudantes universitários. 2016. Tese (Mestrado em psicologia) - Universidade de Aveiro, 2016.

FEHM, L.; SCHMIDT, K. Performance anxiety in gifted adolescent musicians. Journal of Anxiety Disorders, v. 20, n. 1, p. 98-109, 2006.

JARASIUNAITE, G. et al. Biofeedback-assisted relaxation and progressive muscle relaxation potential for enhancing students' distress tolerance. European Scientific Journal, v. 11, n. 2, p. 278-295, 2015.

KASSEL, S. C.; LEMAY, J. Interpersonal Biofeedback. The Therapist, 2012. Available from: http://www.kassel.us/wp-content/uploads/2017/02/Interpersonal_Biofeedback CAMFT_ JF12.pdf. Cited: 22 abr. 2018.

KENNY, D. T. A Systematic review of treatments for music performance anxiety. Anxiety, Stress \& Coping, v. 18, n. 3, p. 183-208, set. 2005.

KENNY, D. T. The factor structure of the revised Kenny Music Performance Anxiety Inventory. Proceedings of the International Symposium on Performance Science, editado por Aaron Williamon, Sharman Pretty e Ralph Buck, p. 37-41. Utrecht: European Association of Conservatoires (AEC), 2009. Available from: http://www.performancescience.org/ ISPS2009/Proceedings/ISPS2009_Proceedings.pdf. Cited: 22 abr. 2018.

KENNY, D. T. The psychology of music performance anxiety. Nova York: Oxford University Press, 2011.

KENNY, D. T. Music performance anxiety: Theory, assessment and treatment. Saarbrücken, Germany: Lambert Academic, 2016.

KESSLER, R. C. et al. Short screening scales to monitor population prevalences and trends in non-specific psychological distress. Psychological Medicine, v. 32, n. 6, p. 959-976, ago. 2002.

LEHMANN, A. C.; SLOBODA, J. A.; WOODY, R. H. Psychology for Musicians: Understanding and acquiring the skills. New York: Oxford University Press, 2007.

OREJUDO, S. et al. The relation of music performance anxiety (MPA) to optimism, self-efficacy, and sensitivity to reward and punishment: Testing Barlow's theory of personal vulnerability on a sample of Spanish music students. Psychology of Music, v. 45, n. 4, p. 570-583, 2017.

OSBORNE, M. S.; KENNY, D. T. The role of sensitizing experiences in music performance anxiety in adolescent musicians. Psychology of Music, v. 36, n. 4, p. 447-462, 10 out. 2008. 
OSBORNE, M. S.; KENNY, D. T.; HOLSOMBACK, R. Assessment of music performance anxiety in late childhood: A validation study of the Music Performance Anxiety Inventory for Adolescents (MPAI-A). International Journal of Stress Management, v. 12, n. 4, p. 312-330, 2005.

PAPAGEORGI, I. The influence of the wider context of learning, gender, age, and individual differences on adolescent musicians' performance anxiety. Proceedings of the International Symposium on Performance Science, editado por Aaron Williamon, Sharman Pretty e Daniela Coimbra, p. 219-224. Utrecht: European Association of Conservatoires (AEC), 2007. Available from: http://www.performancescience.org/ISPS2007/Proceedings/ISPS2007_Proceedings. pdf. Cited: 15 abr. 2018.

PEREIRA, A. et al. Confiabilidade e estrutura fatorial da Escala de Distress Psicológico de Kessler de 10 itens (K10) entre adultos portugueses. Ciência \& Saúde Coletiva, 2017. Disponível em: http://www.cienciaesaudecoletiva.com.br/ artigos/confiabilidade-e-estrutura-fatorial-da-escala-de-distress-psicologico-de-kessler-de-10-itens-k10-entre-adultos-portugueses/16237?id=16237. Acesso em: 14 abr. 2018.

RAY, S. Fatores de estresse e ansiedade na performance musical: Histórico e perspectivas após 10 anos de Simcam. Percepta, v. 1, n. 2, p. 115-126, 2014.

ROCHA, S. F. Ansiedade na performance musical: estudo molecular de associação e validação da escala de "K-MPAl". 2012. Tese (Doutorado em Medicina) - Universidade de São Paulo, Faculdade de Medicina, São Paulo, 2012.
ROCHA, S. F.; DIAS-NETO, Emanuel.; GATTAZ, Wagner Farid. Ansiedade na performance musical: Tradução, adaptação e validação do Kenny music performance anxiety inventory (K-MPAI) para a língua portuguesa. Revista de Psiquiatria Clínica, v. 38, n. 6, p. 217-221, 2011.

SANCHO, L. Estudo da ansiedade em alunos do ensino superior utilizando o Biofeedback. 2013. Tese (Doutorado em psicologia) - Universidade de Aveiro, 2013.

SANTOS, S. C.; SILVA, D. R. Adaptação do state-trait anxiety inventory (STAI) - Form Y para a população portuguesa: primeiros dados. Revista Portuguesa de Psicologia, v. 32, 1997.

SCHWARTZ, M.; ANDRASIK, F. Biofeedback: A practitioner's guide. Quarta edição. Nova York: The Guilford Press, 2016.

SINICO, A.; WINTER, L. Ansiedade na performance musical: causas, sintomas e estratégias de estudantes de flauta. Opus, Porto Alegre, v. 19, n. 1, p. 239-264, 2013.

SPIELBERGER, C. Manual for the State-Trait Anxiety Inventory STAI (form Y) ("Self-Evaluation Questionnaire". Palo Alto: Consulting Psychologists Press, 1983.

THURBER, M. R. et al. Effects of Heart Rate Variability Coherence Biofeedback Training and Emotional Management Techniques to Decrease Music Performance Anxiety. Biofeedback, v. 38, n. 1, p. 28-40, 2010.

WEST, K. Biofeedback. Nova York: Infobase Publishing, 2007. 